\title{
Molecular gas and nuclear activity in early-type galaxies: any link with radio loudness?^
}

\author{
Ranieri D. Baldi ${ }^{1,2,3}$, Marcello Giroletti ${ }^{4}$, Alessandro Capetti ${ }^{5}$, Gabriele Giovanninii ${ }^{4,6}$, Viviana Casasola ${ }^{4}$, \\ Miguel A. Pérez-Torres ${ }^{7}$, and Nario Kuno ${ }^{8}$
}

\author{
1 SISSA-ISAS, via Bonomea 265, 34136 Trieste, Italy \\ 2 Physics Department, The Technion, 32000 Haifa, Israel \\ e-mail: baldi@ph.technion.ac.il \\ 3 Physics Department, Faculty of Natural Sciences, University of Haifa, 32000 Haifa, Israel \\ ${ }^{4}$ INAF-Istituto di Radio Astronomia, via P. Gobetti 101, 40129 Bologna, Italy \\ 5 INAF-Osservatorio Astrofisico di Torino, via Osservatorio 20, 10025 Pino Torinese, Italy \\ 6 Dipartimento di Fisica e Astronomia, Università di Bologna, via Ranzani 1, 40127 Bologna, Italy \\ 7 Instituto de Astrofísica de Andalucía - CSIC, PO Box 3004, 18008 Granada, Spain \\ 8 Division of Physics, Faculty of Pure and Applied Sciences, University of Tsukuba, Japan
}

Received 8 October 2014 / Accepted 2 December 2014

\section{ABSTRACT}

\begin{abstract}
Aims. We want to study the amount of molecular gas in a sample of nearby early-type galaxies (ETGs) that host low-luminosity active galactic nuclei (AGN). We look for possible differences between the radio-loud (RL) and radio-quiet (RQ) AGN.

Methods. We observed the $\mathrm{CO}(1-0)$ and $\mathrm{CO}(2-1)$ spectral lines with the IRAM $30 \mathrm{~m}$ and NRO $45 \mathrm{~m}$ telescopes for eight galaxies. They belong to a large sample of 37 local ETGs that host both RQ and RL AGN. We gather data from the literature for the entire sample.

Results. We report the new detection of $\mathrm{CO}(1-0)$ emission in four galaxies (UGC 0968, UGC 5617, UGC 6946, and UGC 8355) and $\mathrm{CO}(2-1)$ emission in two of them (UGC 0968 and UGC 5617). The $\mathrm{CO}(2-1) / \mathrm{CO}(1-0)$ ratio in these sources is $\sim 0.7 \pm 0.2$. Considering both the new observations and the literature, the detection rate of $\mathrm{CO}$ in our sample is $55 \pm 9 \%$, with no statistically significant difference between the hosts of RL and RQ AGNs. For all the detected galaxies we converted the CO luminosities into the molecular masses, $M_{\mathrm{H}_{2}}$, which range from $10^{6.5}$ to $10^{8.5} M_{\odot}$, without any statistically significant differences between RL and RQ galaxies. This suggests that the amount of molecular gas does not likely set the radio loudness of the AGN. Furthermore, despite the low statistical significance, the presence of a weak trend between the $\mathrm{H}_{2}$ mass with various tracers of nuclear activity (mainly [O III] emission line nuclear power) cannot be excluded.
\end{abstract}

Key words. galaxies: active - galaxies: elliptical and lenticular, cD - galaxies: nuclei - galaxies: ISM - ISM: molecules

\section{Introduction}

Carbon monoxide $\mathrm{CO}$ is the most abundant molecule in galaxies after $\mathrm{H}_{2}\left(\mathrm{CO} / \mathrm{H}_{2} \sim 6 \times 10^{-5}\right)$. It is easy to excite, and it radiates efficiently at frequencies that can be observed fairly easily from the ground. Rotational transitions of $\mathrm{CO}$ are therefore the best tracers of cold molecular gas in galaxies.

In general, early-type galaxies (ETGs) contain an interstellar medium (ISM) with components similar to those found in spiral galaxies as shown by radio, optical, and X-ray observations. However, the detailed analysis of the gas components of large samples of galaxies has shown differences in ISM between galaxies of different morphological types. In addition, the spatial distribution and the relative gas fraction of the different phases differ between early and late-type galaxies. The molecular gas in ETGs seems to be more centrally concentrated than in later types (e.g., Böker et al. 2003; Komugi et al. 2008; Davis et al. 2013). In elliptical galaxies a hot $X$-ray radiating coronal halo is the most massive gaseous component with masses between $10^{8}$

^ Based on observations carried out with the IRAM 30 m telescope. IRAM is supported by INSU/CNRS (France), MPG (Germany), and IGN (Spain). and $10^{10} M_{\odot}$ (e.g., Fabbiano 1989). Warm ionized gas with estimated masses of $10^{3}-10^{5} M_{\odot}$ is found in $\sim 60 \%$ of the observed ellipticals (e.g., Phillips et al. 1986; Sadler 1987; Burstein et al. 1997; Beuing et al. 1999; Bettoni et al. 2003). The presence of a cold gas component in ellipticals has initially been inferred from observations of HI and mid- and far-infrared emission (Knapp et al. 1985, 1989). A growing number of ellipticals are found to contain a molecular gas component with masses ranging from $10^{7}$ to $10^{10} M_{\odot}$ (e.g., Lees et al. 1991; Wiklind et al. 1995; Young 2002, 2005; Bettoni et al. 2003; Combes et al. 2007; Sage et al. 2007; Young et al. 2011; O'Sullivan et al. 2015).

Moreover, since an ETG can host both RL and RQ AGN, this type of galaxy appears to be a critical class of objects where a wide variety of nuclear and host properties might help us to understand their connection. The relative fraction of hot and cold gas phases, traced by the X-ray emitting coronal emission and the CO molecule present in the ISM of ETGs, might play an important role in their accretion mode and their radio loudness. Recently, Werner et al. (2014) have studied the properties of the multiphase gas in ETGs and their role in fueling radio-mode AGN feedback.

To quantify the molecular gas content in the ETGs, we take advantage of the substantial knowledge about the multiband 
nuclear and host properties of a sample of ETGs described below. Capetti \& Balmaverde (2005) studied the optical surface brightness profiles based on the HST images of 37 nearby ETGs, by separating the ETGs on the basis of the inner logarithmic slope $\gamma$ into Core $(\gamma \leq 0.3)$ and Power-law $(\gamma>0.5)$ galaxies (hereafter CoreG and PlawG, respectively). This sample includes both RL and RQ AGNs. On one hand, the CoreG show flat optical core and host RL AGN, with multiwavelength nuclear and host properties similar to those of the FR Is (Fanaroff $\&$ Riley 1974). On the other hand, PlawG are the counterparts of the RQ Seyfert galaxies in ETGs at lower nuclear luminosities with similar sharp inner optical profiles (Capetti \& Balmaverde 2007).

In this work we explore the link between the molecular gas present in these complete samples of ETGs and the radio loudness and the nuclear properties. The structure of this paper is as follows. In Sect. 2, we describe our observations and the literature data we used to complete the sample. In Sect. 3, we present the results giving $\mathrm{H}_{2}$ masses of galaxies and the computed $\mathrm{CO}(2-1) / \mathrm{CO}(1-0)$ ratio. In Sect. 4. we search for empirical relations between the molecular gas and the nuclear activity, considering the entire sample of CoreG and PlawG. In Sect. 5, we summarize the results and draw conclusions.

We adopt a Hubble constant of $H_{0}=71 \mathrm{~km} \mathrm{~s}^{-1} \mathrm{Mpc}^{-1}$ and $q_{0}=0.5$. Assuming a different cosmology would not affect our results since the redshift of the sources considered in this paper is limited to $z=0.01$.

\section{Sample, observations, and data}

The galaxies studied in this work belong to a sample of luminous $(B<14)$, nearby $\left(V_{\text {rec }}<3000 \mathrm{~km} \mathrm{~s}^{-1}\right)$ ETGs. Wrobel \& Heeschen (1991) performed a VLA survey with a flux cutoff of $\sim 1 \mathrm{mJy}$ at $5 \mathrm{GHz}$ on this sample. For the radio-detected objects, Capetti \& Balmaverde (2005) used archival HST observations, which are available for 48 objects, to study their surface brightness profiles and to separate these ETGs following the Nukers scheme (Faber et al. 1997) rather than the traditional morphological classification (i.e., into E and S0 galaxies). They divided the sample in CoreG and PlawG, characterized respectively by a flat and a sharp inner logarithmic slope of their optical brightness profiles. For 37 galaxies, the surface brightness profile is sufficiently well-behaved to yield a successful classification. These sources constitute the sample studied in this work.

Their multiwavelength (radio, optical, X-ray, and emission line) properties have been analyzed in a series of papers by Capetti \& Balmaverde (2005, 2006, 2007), Balmaverde \& Capetti (2006), Balmaverde et al. (2006), Baldi \& Capetti (2009), and Baldi \& Capetti (in prep.). These studies show that the two optical classes of objects correspond to different AGN properties. On one hand, the CoreG represent "miniature radio galaxies", i.e., a scaled-down version of FR Is (Baldi \& Capetti 2009). They invariably host RL nuclei, spectroscopically classified as low-excitation galaxies, with an average radio-loudness parameter of $\mathrm{R}=\mathrm{L}_{5} \mathrm{GHz} / L_{\mathrm{B}} \sim 4000$, similarly to FR Is. In addition, they share with the FR Is a common non-thermal AGN jet origin of the radio, X-ray, and optical nuclear emission (e.g., Chiaberge et al. 1999; Balmaverde \& Capetti 2006). On the other hand, the PlawG are low-luminosity RQ AGNs (on average 100 time less RL than the CoreG), mostly with LINER spectra, but in a few cases they are also associated with Seyfert optical spectra (Baldi \& Capetti, in prep.). They show a deficit in radio luminosity at a given optical and X-ray luminosity with respect to the CoreG (Capetti \& Balmaverde 2006, 2007; Kharb et al. 2012).
Although the sample is detected in the radio band, as well in other wavelengths thanks to deep multiband surveys, the sample is not radio-selected since it includes both RQ and RL AGNs (20 and 17 sources, respectively).

With the Nobeyama Radio Observatory (NRO) $45 \mathrm{~m}$ telescope in Nobeyama (Japan) and with IRAM 30 m telescope in Pico Veleta (Spain) we proposed to observe the sources of the sample that do not have previous $\mathrm{CO}$ detections. Here we report the $\mathrm{CO}$ data for the eight sources for which we got observing time in April and October-November 2007, respectively for the NRO and IRAM telescopes. Four galaxies were observed with the NRO telescope covering the $\mathrm{CO}(1-0)$ line at $115 \mathrm{GHz}$ and five with the IRAM $30 \mathrm{~m}$ telescope to detect the $\mathrm{CO}(1-0)$ line, as well as the $\mathrm{CO}(2-1)$ line at $230 \mathrm{GHz}$. UGC 6946 was observed with both telescopes. The beam full width at half maximum (FWHM) of NRO at $115 \mathrm{GHz}$ is $15^{\prime \prime}$, while those of the IRAM 30 m telescope are $22^{\prime \prime}$ and $13^{\prime \prime}$ respectively, at the two $\mathrm{CO}$ lines frequencies. The observations at NRO were carried out with $\mathrm{S} 100$ receiver and with auto correlator (AC) spectrometer with a band width of $512 \mathrm{MHz}$, whereas those at the IRAM $30 \mathrm{~m}$ telescope using the wobbler switching mode with band widths of 512 and $1024 \mathrm{MHz}$ for the two frequencies.

For NRO we spent about five hours for a single object to reach a root mean square (rms) noise of $\sim 3 \mathrm{mK}$ for $\sim 30 \mathrm{~km} \mathrm{~s}^{-1}$ channel. Instead the total integration time for each source observed with the IRAM $30 \mathrm{~m}$ telescope was about one to three hours, resulting in a relatively homogeneous level of noise of $2 \mathrm{mK}$ per 15 and $30 \mathrm{~km} \mathrm{~s}^{-1}$ channel, respectively, for the two $\mathrm{CO}$ frequencies. The signal is expressed in main beam temperature $T_{\mathrm{mb}}$, since the sources are not expected to be extended, and homogeneous. The parameter $T_{\mathrm{mb}}$ is related to the equivalent antenna temperature reported above the atmosphere $\left(T_{\mathrm{A}}^{*}\right)$ by $\eta=T_{\mathrm{A}}^{*} / T_{\mathrm{mb}}$, where $\eta$ is the telescope main-beam efficiency. For IRAM telescope, at $115 \mathrm{GHz} \eta=0.79$ and at $230 \mathrm{GHz}$ $\eta=0.54$, while $\eta=0.34$ at $115 \mathrm{GHz}$ for the NRO telescope. Each spectrum was summed and reduced using linear baselines.

\section{Results}

\subsection{CO spectra}

We now present the new $\mathrm{CO}$ observations of the eight objects. Figure 1 shows the NRO spectra for the $\mathrm{CO}(1-0)$ rotational transition for four objects, and Fig. 2 shows the IRAM spectra for both the $\mathrm{CO}(1-0)$ and $\mathrm{CO}(2-1)$ lines for five objects. We detect the $\mathrm{CO}(1-0)$ and $\mathrm{CO}(2-1)$ lines for four (out of 9) and two (out of 5) of the observed objects, respectively.

Since some lines have complex profiles, to yield a correct measurement of the flux for the $3 \sigma$ detected line, we directly integrate the $T_{\mathrm{mb}}$ profile on the spectrum for all the sources:

$I_{\mathrm{CO}}=\int T_{\mathrm{mb}}(v) \mathrm{d} v \quad\left[\mathrm{~K} \mathrm{~km} \mathrm{~s}^{-1}\right]$.

For the undetected CO lines, assuming a typical $250 \mathrm{~km} \mathrm{~s}^{-1}$ line width (e.g., Young et al. 2011), corresponding to a good sampling of the line with at least eight (16) points in the spectrum with 30 (15) $\mathrm{km} \mathrm{s}^{-1}$ of spectral resolution for NRO (IRAM) telescope, the $1 \sigma$ upper limits are evaluated in this way:

$I_{\mathrm{CO}}=\sqrt{N_{\mathrm{ch}}} \mathrm{rms}_{T_{\mathrm{mb}}} \Delta v$

where $N_{\mathrm{ch}}$ is the number of channel covered in $250 \mathrm{~km} \mathrm{~s}^{-1}$ line width $(\Delta v)$, and $\mathrm{rms}_{T_{\mathrm{mb}}}$ is the temperature rms of the spectrum.

Among the four sources showing $\mathrm{CO}(1-0)$ detection, UGC 0968 and UGC 6946 exhibit double-horn profiles, 
R. D. Baldi et al.: Molecular gas and nuclear activity in early-type galaxies: any link with radio loudness?
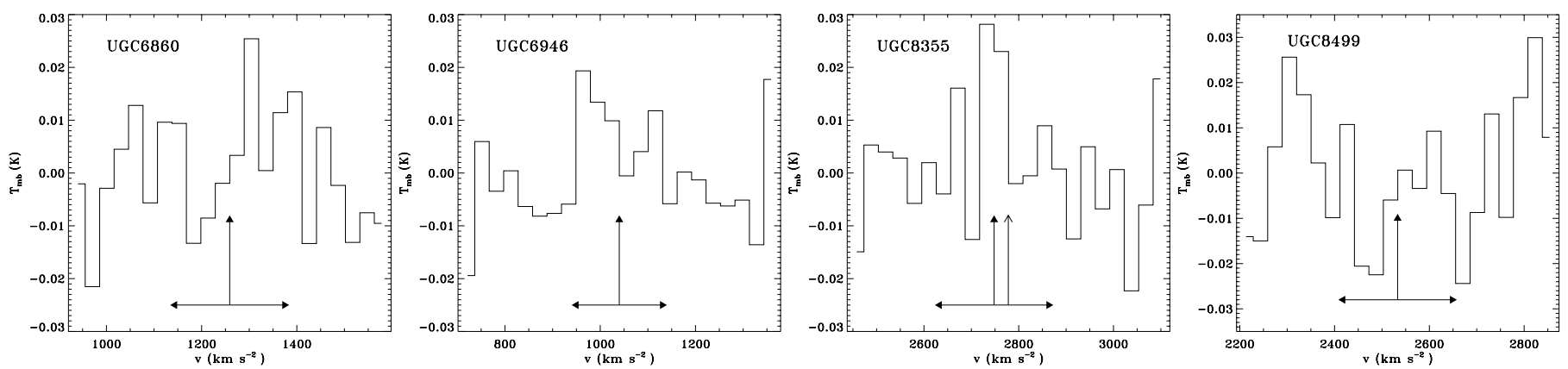

Fig. 1. $\mathrm{CO}(1-0)$ spectra of the objects observed with the NRO telescope. In each panel, the vertical arrow with filled head represents the central position of the line used to perform the Gaussian fit, while the other arrow marks the position expected by galaxy's recession velocity. The horizontal arrows represent the velocity range on which the spectrum is integrated to derive the flux measurement.

Table 1. Results of the CO observations on our sample of ETGs.

\begin{tabular}{|c|c|c|c|c|c|c|c|c|c|c|c|}
\hline \multirow[b]{2}{*}{ Galaxy name } & \multirow[b]{2}{*}{$\begin{array}{c}V \\
\mathrm{~km} \mathrm{~s}^{-1} \\
\end{array}$} & \multirow[b]{2}{*}{ Telescope } & \multicolumn{3}{|c|}{${ }^{12} \mathrm{CO}(1-0)$} & \multirow[b]{2}{*}{ Profile } & \multicolumn{2}{|c|}{${ }^{12} \mathrm{CO}(2-1)$} & \multirow[b]{2}{*}{$\begin{array}{l}\mathrm{rms} \\
\mathrm{mK}\end{array}$} & \multirow[b]{2}{*}{$\begin{array}{c}M_{\mathrm{H}_{2}} \\
10^{8} M_{\odot} \\
\end{array}$} & \multirow[b]{2}{*}{$\mathrm{CO}$ ratio } \\
\hline & & & $\begin{array}{c}F_{\mathrm{CO}} \\
\mathrm{K} \mathrm{km} \mathrm{s}{ }^{-1}\end{array}$ & $\begin{array}{c}\Delta v \\
\mathrm{~km} \mathrm{~s}^{-1}\end{array}$ & $\begin{array}{l}\mathrm{rms} \\
\mathrm{mK}\end{array}$ & & $\begin{array}{c}F_{\mathrm{CO}} \\
\mathrm{K} \mathrm{km} \mathrm{s}^{-1}\end{array}$ & $\begin{array}{l}\Delta v \\
\mathrm{~s}^{-1}\end{array}$ & & & \\
\hline UGC 0968 & 2379 & IRAM & $3.45 \pm 0.58$ & 430 & 7.2 & $\mathrm{dh}$ & $5.9 \pm 1.2$ & 430 & 10 & $1.48 \pm 0.25$ & $0.60 \pm 0.2$ \\
\hline UGC 5617 & 1151 & IRAM & $1.40 \pm 0.40$ & 304 & 5.9 & $\mathrm{sp}$ & $3.03 \pm 0.37$ & 300 & 3.8 & $0.31 \pm 0.09$ & $0.75 \pm 0.2$ \\
\hline UGC 6860 & 1259 & NRO & $<9.4$ & - & 11.0 & & & & & $<0.85$ & \\
\hline UGC 6946 & 1040 & IRAM & $<4.8$ & - & 4.1 & & $<1.2$ & - & 3.7 & $<0.71$ & \\
\hline UGC 6946 & 1040 & NRO & $2.5 \pm 0.7$ & 187 & 9.0 & dh & & & & $0.17 \pm 0.05$ & \\
\hline UGC 7203 & 2215 & IRAM & $<4.5$ & - & 4.5 & & $<1.4$ & - & 4.6 & $<2.95$ & \\
\hline UGC 8355 & 2778 & NRO & $2.3 \pm 0.7$ & 240 & 8.2 & $\mathrm{sp}$ & & & & $0.91 \pm 0.28$ & \\
\hline UGC 8499 & 2533 & NRO & $<10.5$ & - & 14.9 & & & & & $<3.6$ & \\
\hline UGC 9706 & 1714 & IRAM & $<4.3$ & - & 3.7 & & $<2.9$ & - & 7.3 & $<1.6$ & \\
\hline
\end{tabular}

Notes. Column description: (1) name; (2) recession velocity; (3) telescope used for the observation; $\operatorname{CO}(1-0)$ observations: (4) integrated line emission in $\mathrm{K} \mathrm{km} \mathrm{s}^{-1}$; (5) velocity width; (6) rms of the spectrum in $\mathrm{mK}$; (7) shape of the line profile: dh (double-horn), sp (single peak); $\mathrm{CO}(2-1)$ observations: (8) integrated line emission in $\mathrm{K} \mathrm{km} \mathrm{s}^{-1}$; (9) spectral region of integration; (10) rms of the spectrum in mK; (11) evaluation of $\mathrm{H}_{2}$ mass from $\mathrm{CO}(1-0)$ line intensity; (12) $\mathrm{CO}(2-1) / \mathrm{CO}(1-0)$ ratio.

while UGC 5617 and UGC 8355 have single-peak profiles. Galaxies detected in the $\mathrm{CO}(2-1)$ line (UGC 0968 and UGC 5617) have profiles similar to those found in the $\mathrm{CO}(1-0)$ line. In Table 1 , we collect the $\mathrm{CO}(1-0)$ and $\mathrm{CO}(2-1)$ line properties for the observed objects.

Among the objects of the sample, a few have additional CO measurement in the literature. For UGC 5617, Lisenfeld et al. (2008) measured both the CO lines with IRAM telescope: $I_{\mathrm{CO}(1-0)}=1.3 \mathrm{~K} \mathrm{~km} \mathrm{~s}^{-1}$, consistent with our IRAM CO flux and $I_{\mathrm{CO}(2-1)}=0.7 \mathrm{~K} \mathrm{~km} \mathrm{~s}^{-1}$, which are slightly smaller than our measurement. Our nondetections of CO(1-0) for UGC 8499 and UGC 9706 are also consistent with the upper limits reported by Combes et al. (2007) with the IRAM telescope, $<2.37$ and $<4.8 \mathrm{~K} \mathrm{~km} \mathrm{~s}^{-1}$, respectively.

\section{2. $\mathrm{CO}$ emission and $\mathrm{H}_{2}$ mass estimation}

From standard, empirical calibrations, it is possible to deduce the interstellar $\mathrm{H}_{2}$ mass content from the integrated CO intensity $I_{\mathrm{CO}}$ $\left(\mathrm{K} \mathrm{km} \mathrm{s}^{-1}\right)$. We adopt here the conversion factor commonly used for

$N\left(\mathrm{H}_{2}\right)=2.3 \times 10^{20} I_{\mathrm{CO}}$

in molecule $/ \mathrm{cm}^{2}$ unit. From this equation, the mass of cold molecular hydrogen contained in one beam of the telescope is

$M_{\mathrm{H}_{2}}=\alpha I_{\mathrm{CO}} A_{\text {beam }}$ where $\alpha$ is the conversion factor, whose commonly used value is $4.8 M_{\odot}\left(\mathrm{K} \mathrm{km} \mathrm{s}^{-1} \mathrm{pc}^{2}\right)^{-1}$ (Solomon \& Barrett 1991), derived from galactic molecular cloud observations, and $A_{\text {beam }}$ is the beam area at the galaxy in $\mathrm{pc}^{2}$.

The conversion between $\mathrm{CO}$ and $M_{\mathrm{H}_{2}}$ column densities and masses remains controversial. Its value varies with the physical gas conditions, metallicity, and environmental factors (e.g., Maloney 1990; Israel 1997; Boselli et al. 2002; Bolatto et al. 2008; Glover \& Mac Low 2011; Magrini et al. 2011) and can change by a factor 4-15 (e.g., Wilson \& Colbert 1995; Casasola et al. 2007). The conversion $\mathrm{H}_{2}-\mathrm{CO}$ value also changes with galaxy morphological type: galaxies earlier than Scd type usually show values comparable to, or lower than, the Galactic one, while extremely late-type spirals or irregular galaxies tend to show higher values (Arimoto et al. 1996).

The $\mathrm{H}_{2}$ can be underestimated if the molecular cloud is more extended than the beam area covered on the galaxy. In our case such a physical region at the distance of the sources corresponds to an aperture that ranges from 0.5 to $4 \mathrm{kpc}$ for the $\mathrm{CO}(1-0)$ transition. CO interferometric observations show that the molecular gas in ETGs is centrally concentrated in the central kiloparsecs (e.g., Braine et al. 1993; Boselli et al. 2002; Böker et al. 2003; Komugi et al. 2008; Davis et al. 2013). Recently by comparing the CO fluxes recovered from $30 \mathrm{~m}$ spectra and from full interferometric maps (from CARMA) for a sample of local ETGs, Alatalo et al. (2013) found that up to half of the molecular mass of a source (at a similar distance to our sample) measured with the $30 \mathrm{~m}$ telescope can be missed. However, no large differences 

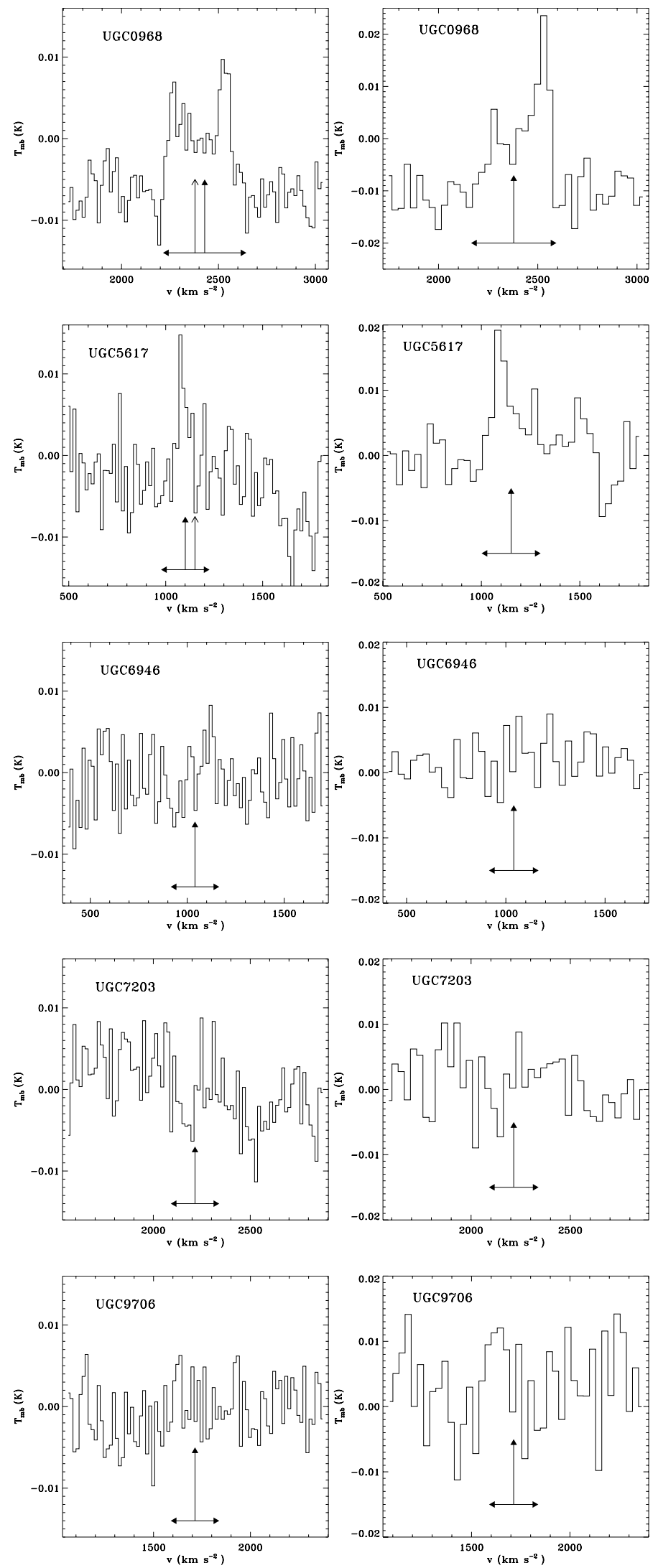

Fig. 2. $\mathrm{CO}(1-0)($ left $)$ and $\mathrm{CO}(2-1)$ (right) spectra of the objects observed with the IRAM $30 \mathrm{~m}$ telescope. In each panel, the vertical arrow with filled head represents the central position of the line used to perform the Gaussian fit, while the other arrow marks the position expected by galaxy's recession velocity. The horizontal arrows represent the velocity range on which the spectrum is integrated to derive the flux measurement. are expected between the $30 \mathrm{~m}$ and $45 \mathrm{~m}$ telescope observations. Therefore, this effect should not significantly affect our results.

With the method explained above, the $\mathrm{H}_{2}$ masses estimated for the eight galaxies are $7.2 \lesssim \log M_{\mathrm{H}_{2}} / M_{\odot} \lesssim 8.5$, and all values are listed in Table 2, including the $\mathrm{CO}$ upper limits.

\section{3. $\mathrm{CO}(2-1) / \mathrm{CO}(1-0)$ ratio}

The $\mathrm{CO}(2-1) / \mathrm{CO}(1-0)$ is measured by the ratio between the integrated emission of the two lines on equal regions of the galaxy. Because of the different beam sizes at 115 and $230 \mathrm{GHz}$, a beamdilution factor is necessary to correct the different spatial resolution, which corresponds to $\sim 4$, the ratio of the beam areas for the two lines, assuming that the gas distribution of both $\mathrm{CO}(3-2)$ and $\mathrm{CO}(1-0)$ is compact. Therefore the $\mathrm{CO}(1-0)$ lines intensities have been multiplied by this beam-correcting factor to be compared to the $\mathrm{CO}(2-1)$ lines. It is possible to evaluate this ratio only for two objects, UGC 0968 and UGC 5617 (Table 1). For UGC 0968 the assumption of a compact CO distribution is less obvious, since one peak of the $\mathrm{CO}(2-1)$ horn profile is less pronounced than in $\mathrm{CO}(1-0)$. This might suggest that the $\mathrm{H}_{2}$ emitting region lies somewhere between the two beam sizes. However, both values are consistent within the uncertainties with the $\mathrm{CO}(2-1) / \mathrm{CO}(1-0)$ line ratio $(\sim 0.6)$ found for samples of radio galaxies (Ocaña Flaquer et al. 2010) and Seyfert galaxies (Papadopoulos \& Seaquist 1998).

\section{4. $\mathrm{H}_{2}$ mass for the sample of nearby ETGs}

From the literature we collect the $\mathrm{H}_{2}$ masses, estimated from $\mathrm{CO}(1-0)$ observations, of the remaining objects of the sample studied by Capetti \& Balmaverde (2006). Combining our new results and the literature data, we now have $\mathrm{CO}$ observations for all but four (out of 37) sources. Table 2 shows the values of molecular masses and the appropriate references for the entire samples of CoreG and PlawG.

The overall CO detection rate is $55 \pm 9 \%$, and it is similar for the two classes of brightness profiles $(50 \pm 12 \%$ for the CoreG and $59 \pm 12 \%$ for the PlawG, respectively). The distributions of the molecular masses for the two subsamples (left panel in Fig. 3) is $10^{6.5}-10^{8.5} M_{\odot}$. This broad range is consistent with the molecular masses found in other samples of ETGs, on average, of several $10^{7} M_{\odot}$ (e.g., Lees et al. 1991; Wiklind et al. 1995; Combes et al. 2007; Crocker et al. 2011; Young et al. 2011).

Given the presence of nondetections in the data, we use the Kaplan-Meier product-limit estimator (Kaplan \& Meier 1958), which provides the mean values for distribution of $M_{\mathrm{H}_{2}}$ with the assumption that the censoring is random, a condition that is fulfilled in our data. For CoreG, $\left\langle\log M_{\mathrm{H}_{2}}\right\rangle=(7.4 \pm 0.2) M_{\odot}$ and for PlawG, $\left\langle\log M_{\mathrm{H}_{2}}\right\rangle=(7.5 \pm 0.2) M_{\odot}$. The two average values are consistent with each other within the errors.

To assess the presence of significantly statistical difference between the $M_{\mathrm{H}_{2}}$ distributions of CoreG and PlawG, we used the "twosampt" task of the Astronomy Survival Analysis (ASURV) package (Lavalley et al. 1992). This package is available under IRAF/STSDAS and provides us with a tool to deal with censored data (Feigelson \& Nelson 1985; Isobe et al. 1986). In particular, the Peto-Prentice test (Peto \& Peto 1972) quantifies the distance between the empirical distribution functions of two samples, with the null hypothesis that they are drawn from the same distribution. The result of the test is that the $M_{\mathrm{H}_{2}}$ distribution of CoreG and PlawG are not drawn from different parent populations with a confidence level greater than $95 \%$. 
Table 2. Multiwavelength properties of the whole sample.

\begin{tabular}{|c|c|c|c|c|c|c|c|c|c|}
\hline Name & $z$ & $M_{\mathrm{H}_{2}}$ & Ref. & $L_{\mathrm{r}}$ & $L_{\mathrm{o}}$ & $L_{\mathrm{X}}$ & $\left.L_{[\mathrm{O}} \mathrm{III}\right]$ & Class & Dust \\
\hline UGC 0968 & 0.007935 & 8.17 & this work & 36.96 & $<39.77$ & - & $<37.75$ & CoreG & $\mathrm{Y}$ \\
\hline UGC 5902 & 0.003039 & $<6.70$ & $\mathrm{CO} 07$ & 35.83 & $<39.10$ & $<38.40$ & 37.96 & CoreG & $\mathrm{N}$ \\
\hline UGC 6297 & 0.003202 & 8.37 & WE03 & 36.84 & $<39.06$ & $<38.40$ & 38.21 & CoreG & $\mathrm{Y}$ \\
\hline UGC 7203 & 0.007388 & $<8.47$ & this work & 37.44 & 39.85 & $<38.93$ & 38.08 & CoreG & $\mathrm{N}$ \\
\hline UGC 7360 & 0.007465 & $<7.84$ & OC 10 & 39.22 & 39.71 & 40.95 & 38.91 & CoreG & $\mathrm{N}$ \\
\hline UGC 7386 & 0.002165 & 7.36 & $\mathrm{CO} 07$ & 38.38 & 38.76 & 39.72 & 38.95 & CoreG & $\mathrm{N}$ \\
\hline UGC 7494 & 0.003536 & $<7.51$ & WI95 & 38.57 & 39.27 & 39.30 & 38.20 & CoreG & $\mathrm{N}$ \\
\hline UGC 7629 & 0.003326 & 7.60 & HU94 & 36.73 & 38.79 & 38.23 & 37.40 & CoreG & $\mathrm{Y}$ \\
\hline UGC 7654 & 0.004360 & 7.72 & OC10 & 39.90 & 40.72 & 40.30 & 38.99 & CoreG & $\mathrm{N}$ \\
\hline UGC 7760 & 0.001134 & $<7.23$ & $\mathrm{CO} 07$ & 37.30 & 38.73 & 38.40 & 37.28 & CoreG & $\mathrm{Y}$ \\
\hline UGC 7797 & 0.006605 & 7.96 & SO93 & 38.05 & $<40.19$ & - & 38.79 & CoreG & $\mathrm{Y}$ \\
\hline UGC 7878 & 0.003129 & $<6.85$ & SA07 & 36.90 & 39.07 & $<38.41$ & 37.89 & CoreG & $\mathrm{Y}$ \\
\hline UGC 7898 & 0.003726 & 7.80 & SA89 & 37.46 & $<39.13$ & $<38.52$ & $<37.34$ & CoreG & $\mathrm{N}$ \\
\hline UGC 8745 & 0.005851 & - & & 37.81 & - & - & 38.41 & CoreG & $\mathrm{Y}$ \\
\hline UGC 9655 & 0.006578 & $<7.46$ & $\mathrm{CO} 07$ & 36.96 & - & - & $<38.04$ & CoreG & $\mathrm{Y}$ \\
\hline UGC 9706 & 0.005717 & $<8.20$ & this work & 37.31 & 39.25 & 38.26 & 38.18 & CoreG & $\mathrm{Y}$ \\
\hline UGC 9723 & 0.002242 & 7.92 & TA94 & 36.92 & - & $<38.18$ & 37.33 & CoreG & $\mathrm{Y}$ \\
\hline UGC 5617 & 0.003839 & 7.49 & this work & 36.88 & $<40.51$ & 40.30 & 38.75 & PlawG & $\mathrm{Y}$ \\
\hline UGC 5663 & 0.004383 & $<8.31$ & SA89 & 36.87 & 40.57 & 38.80 & 38.59 & PlawG & $\mathrm{Y}$ \\
\hline UGC 5959 & 0.004717 & $<6.95$ & $\mathrm{CO} 07$ & 37.16 & 40.54 & 39.80 & 38.77 & PlawG & $\mathrm{Y}$ \\
\hline UGC 6153 & 0.008836 & 8.61 & MA97 & 38.14 & 42.11 & 42.40 & 40.80 & PlawG & $\mathrm{Y}$ \\
\hline UGC 6860 & 0.004200 & $<7.93$ & this work & 36.38 & $<40.15$ & 39.60 & 38.28 & PlawG & $\mathrm{N}$ \\
\hline UGC 6946 & 0.003469 & 7.24 & this work & 38.16 & 41.40 & 41.00 & 39.42 & PlawG & $\mathrm{Y}$ \\
\hline UGC 6985 & 0.003102 & 7.94 & WE03 & 36.31 & $<40.56$ & - & $<37.51$ & PlawG & $\mathrm{N}$ \\
\hline UGC 7005 & 0.004820 & - & & 36.94 & $<40.37$ & 40.40 & 39.09 & PlawG & $\mathrm{Y}$ \\
\hline UGC 7103 & 0.002692 & 7.20 & WE03 & 36.39 & 40.85 & 39.40 & 38.45 & PlawG & $\mathrm{N}$ \\
\hline UGC 7142 & 0.003196 & $<6.65$ & WE03 & 36.96 & 40.44 & 39.90 & 38.71 & PlawG & $\mathrm{Y}$ \\
\hline UGC 7256 & 0.003623 & 6.88 & WE03 & 37.33 & 40.57 & 40.20 & 39.01 & PlawG & $\mathrm{Y}$ \\
\hline UGC 7311 & 0.007909 & - & & 37.07 & - & - & 38.35 & PlawG & $\mathrm{Y}$ \\
\hline UGC 7575 & 0.002672 & 7.98 & VO05 & 36.00 & - & 39.30 & 38.72 & PlawG & $\mathrm{Y}$ \\
\hline UGC 7614 & 0.004036 & 8.23 & $\mathrm{CO} 07$ & 36.15 & 40.47 & $<40.40$ & $<37.85$ & PlawG & $\mathrm{Y}$ \\
\hline UGC 8355 & 0.009267 & 7.96 & this work & 36.69 & 41.27 & - & $<38.31$ & PlawG & $\mathrm{N}$ \\
\hline UGC 8499 & 0.008449 & $<8.56$ & this work & 37.09 & $<40.37$ & - & 38.31 & PlawG & $\mathrm{N}$ \\
\hline UGC 8675 & 0.003549 & $<7.90$ & MA97 & 36.34 & 40.33 & 40.60 & 39.66 & PlawG & $\mathrm{Y}$ \\
\hline UGC 9692 & 0.004533 & $<7.34$ & $\mathrm{CO} 07$ & 36.65 & $<40.41$ & - & 37.92 & PlawG & $\mathrm{Y}$ \\
\hline UGC 10656 & 0.009306 & - & & 37.05 & $<40.96$ & 40.10 & 38.90 & PlawG & $\mathrm{N}$ \\
\hline UGC 12759 & 0.005704 & 8.12 & MA97 & 36.93 & 40.49 & 41.47 & 39.51 & PlawG & $\mathrm{N}$ \\
\hline
\end{tabular}

Notes. Column description: (1) name; (2) redshift; (3) $\mathrm{H}_{2}$ mass in $M_{\odot}$ and references (4): CO07 Combes et al. (2007), WE03 Welch \& Sage (2003), OC10 Ocaña Flaquer et al. (2010), WI95 Wiklind et al. (1995), HU94 Huchtmeier et al. (1994), SO93 Sofue \& Wakamatsu (1993), SA07 Sage et al. (2007), SA89 Sage \& Wrobel (1989), TA94 Taniguchi et al. (1994), MA97 Maiolino et al. (1997), VO05 Vollmer et al. (2005); nuclear luminosities [erg s ${ }^{-1}$ from Balmaverde \& Capetti (2006) and Capetti \& Balmaverde (2006) in (5) radio, (6) optical, and (7) and X-ray (2-10 keV); (8) [O III] emission line luminosity [erg s ${ }^{-1}$ ] from Capetti \& Balmaverde (2006), Baldi \& Capetti (2009); (9) optical profile; (10) presence of dust structure in HST optical image? Y for yes and $\mathrm{N}$ for no.

In Fig. 4 we compare the molecular masses and stellar luminosity, $L_{*}$, estimated from the $K$-band absolute magnitude of the galaxy. The contribution of molecular mass is in the range $\sim 10^{-2}-10^{-4}$ of the galaxy luminosity (corresponding to the galaxy mass). Capetti \& Balmaverde (2006) find that the CoreG hosts are more luminous and so more massive than those of the PlawG. Nevertheless, the Peto-Prentice test keeps on confirming that the CoreG and PlawG are not significantly different in terms of fraction $M_{\mathrm{H}_{2}} / L_{*}$, with a confidence level greater than $95 \%$.

We further investigated the presence of a difference between CoreG and PlawG in the HST optical images (Capetti \& Balmaverde 2005) to look for an association between the presence of dust and the $\mathrm{CO}$ detection. We excluded the four objects (UGC 7005, UGC 7311, UGC 8745, and UGC 10656) that have no $\mathrm{CO}$ observations (Table 2). Most of the objects (20/33) show evident optical obscuration in the nuclear region. Among the 20 objects with dust present in their hosts,
11 have a $\mathrm{CO}$ detection. Among the 13 objects without dust structure, 7 have a $\mathrm{CO}$ detection. This implies that there is no clear association between the presence of dust and the $\mathrm{CO}$ detection in the ETGs of our sample. In addition, the dust is indifferently present in CoreG and PlawG.

\subsection{Molecular gas and nuclear properties}

Another approach to studing the molecular gas content in our sample of ETGs is to look for a possible connection with their nuclear properties by considering the multiwavelength emission from their AGN. Effectively, we compare $M_{\mathrm{H}_{2}}$ with various tracers of nuclear activity, in terms of radio (at $5 \mathrm{GHz}$ ), optical, $\mathrm{X}$-ray (2-10 keV), and [O III] emission line nuclear power for the entire sample (see panels in Fig. 5).

At a first glance, the panels of Fig. 5 show "trends", with relatively large scatters, between the $\mathrm{H}_{2}$ mass and different indicators of the AGN power. Our new $\mathrm{CO}$ measurements are, 

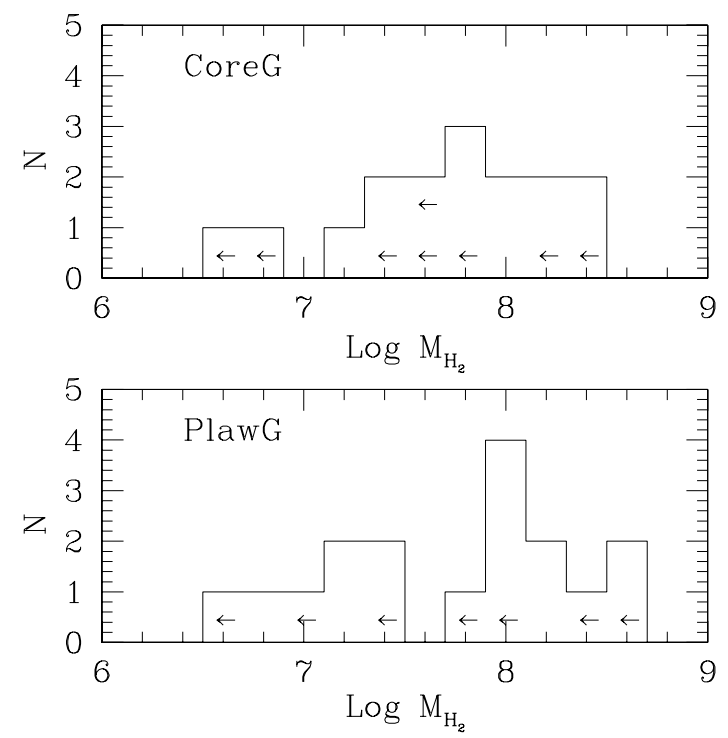

Fig. 3. Histograms of the $M_{\mathrm{H}_{2}}$ in $M_{\odot}$ units for the CoreG (top panel) and PlawG (bottom panel). The arrows indicate the upper limits on the molecular mass measurement.

on average, located at high molecular masses and intermediate nuclear luminosities. However, it is interesting to point out that UGC 6946, which has the lowest $\mathrm{H}_{2}$ mass among the new $\mathrm{CO}$ detection, is one of the most luminous sources in [O III], X-ray, optical, and radio of the sample.

To assess the presence of correlations, we performed a statistical censored analysis using the ASURV package. The method we use is based on the assumption that the censored data are uniformly distributed, a condition that is satisfied for our data. We used the "schmittbin" task (Schmitt 1985) to calculate the associated linear regression coefficients for two sets of variables. Operatively, we carried out this procedure twice, obtaining two linear regressions: first, we considered the former quantity as the independent variable and the latter as the dependent one; second, we switched the roles of the variables. The best fit is represented by the bisector of these two regression lines. This follows the suggestion of Isobe et al. (1990) who consider such a method preferable because of problems that require a symmetrical treatment of the two variables. To estimate the quality of the linear regression, we derived the generalized Kendall's $\tau$ (Kendall 1983) between the two variables, using the "bhkmethod" task. The statistical parameters of all the linear regressions are reported in Table 3.

Although the low statistical parameters, the presence of a weak trend between the [O III] line luminosity and $M_{\mathrm{H}_{2}}$ (upper left panel of Fig. 5) can be marginally considered. The Kendall's $\tau$ coefficient is 0.140 with an associated probability that a fortuitous correlation appears at a level measured by our test statistic of 0.42 . For the other tracers of the nuclear activity, X-ray, optical, and radio band (right upper panel and lower panels of Fig. 5), the statistical significance of a relation with the molecular gas mass is lower. In fact, the probability that a fortuitous relation appears in those plots are $0.65,0.69$, and 0.67 , respectively (Table 3 ). In the $L_{\text {radio }}-M_{\mathrm{H}_{2}}$ panel, the lack of the relation is also probably driven by the different radio loudness of CoreG and PlawG. We cannot establish the type of relation between $L_{\mathrm{AGN}}$ and $M_{\mathrm{H}_{2}}$ since the slopes of the trends are not constrained well owing to the small sample and the large scatter.

Although caution is advised, the significant scatter observed in the observed trends $M_{\mathrm{H}_{2}}-L_{\mathrm{AGN}}$ suggests that, if a connection

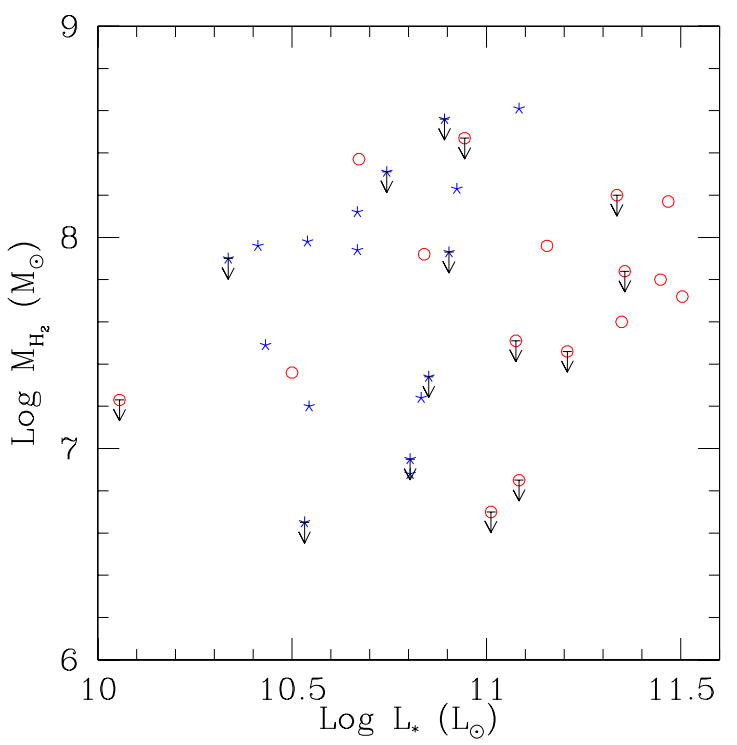

Fig. 4. Luminosity of the host galaxies estimated from the $K$-band magnitude (in $L_{\odot}$ units) vs. $\mathrm{H}_{2}$ masses (in $M_{\odot}$ units). The RL AGN (CoreG) are the red empty circles, while the RQ AGN (PlawG) are the blue stars.

between nuclear properties and molecular gas content is present, it is certainly affected by complex mechanisms. The lack of clear starburst regions in the optical HST images (Capetti \& Balmaverde 2005) and no evidence of young stellar populations in their optical spectra (Baldi \& Capetti 2009) rule out a star formation origin for these trends. A reasonable, but not obvious, explanation of the trends might be the most basic mechanism of heating of the molecular clouds by high-energy photons (e.g., UV and X-ray photons) from the AGN, as suggested by Braine $\&$ Combes (1992). The increase in the AGN luminosity might account for the increase in the CO luminosity, i.e., the molecular mass heated by the radiation field and, then, emitting CO lines. The possible sources of the production of high-energy photons are the accretion disk and the relativistic radio jet. The contribution of the former is important for the (RQ) PlawG, and the latter for the (RL) CoreG (Capetti \& Balmaverde 2006; Balmaverde $\&$ Capetti 2006). This explanation is in line with the results of Nesvadba et al. (2010) and Ogle et al. (2010) for RL AGN, which is consistent with a model of cold gas emission powered by the dissipation of mechanical energy through shocks by radio jets.

\section{Summary and conclusions}

We have analyzed the $\mathrm{CO}(1-0)$ and $\mathrm{CO}(2-1)$ transition spectra for eight nearby galaxies observed with NRO and IRAM telescopes. These sources belong to a sample of 37 ETGs whose nuclear and host properties are well studied. They host the lowluminosity RL and RQ AGN associated with flat (CoreG) or sharp (PlawG) optical profile, respectively. We converted the $\mathrm{CO}(1-0)$ luminosities in $\mathrm{H}_{2}$ mass using standard correction factors. To complete the sample, we found in the literature the molecular masses for the remaining of the sample, ranging from $10^{6.5}$ to $10^{8.5} M_{\odot}$.

Even though they differ in nuclear and host properties, we did not find any statistical significant difference in terms of molecular gas content between CoreG and PlawG. This suggests that the amount of molecular gas does not seem to be related to the radio loudness of the AGN in an ETG. Since singledish observations do not provide any spatial information about the molecular gas distribution, it is reasonable to propose that 
R. D. Baldi et al.: Molecular gas and nuclear activity in early-type galaxies: any link with radio loudness?
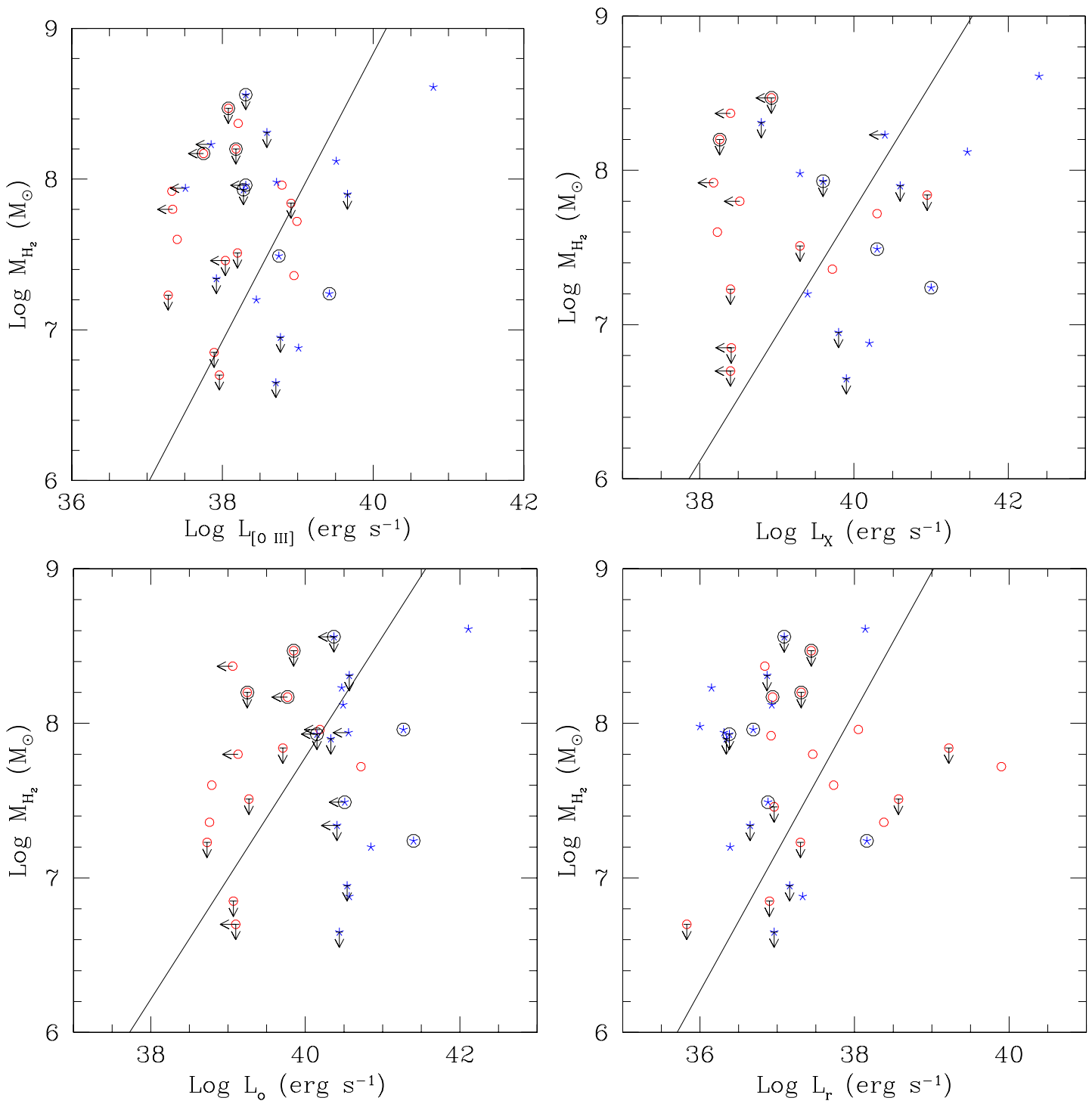

Fig. 5. Multiwavelength luminosities (in $\mathrm{erg} \mathrm{s}^{-1}$ ) versus $\mathrm{H}_{2}$ masses (in $M_{\odot}$ ). Upper left panel: [O III] luminosities vs. $M_{\mathrm{H}_{2}}$; upper right panel: nuclear X-ray luminosities vs. $M_{\mathrm{H}_{2}}$; lower left panel: optical luminosities vs. $M_{\mathrm{H}_{2}}$; lower right panel radio core luminosities (5 GHz) vs. $M_{\mathrm{H}_{2}}$. The RL AGN (CoreG) are the red empty circles, while the RQ AGN (PlawG) are the blue stars. The large black circles mark the molecular masses estimated from our CO observations.

Table 3. Summary of the statistical analysis of the linear regressions for the sample.

\begin{tabular}{c|c|cc|cc|cc}
\hline \hline $\begin{array}{c}\text { Relation } \\
(1)\end{array}$ & $\begin{array}{c}\text { Class } \\
(2)\end{array}$ & $\begin{array}{c}N_{d} \\
(3)\end{array}$ & $\begin{array}{c}N_{c} \\
(4)\end{array}$ & $\begin{array}{c}\tau \\
(5)\end{array}$ & $\begin{array}{c}P_{\tau} \\
(6)\end{array}$ & $\begin{array}{c}m \\
(7)\end{array}$ & $\begin{array}{c}q \\
(8)\end{array}$ \\
\hline$L_{[\mathrm{O} \mathrm{III}}-M_{\mathrm{H}_{2}}$ & all & 13 & 20 & 0.140 & 0.416 & $0.95 \pm 0.30$ & $-29 \pm 17$ \\
$L_{\mathrm{X}}-M_{\mathrm{H}_{2}}$ & all & 11 & 15 & 0.092 & 0.647 & $0.82 \pm 0.40$ & $-25 \pm 20$ \\
$L_{\mathrm{O}}-M_{\mathrm{H}_{2}}$ & all & 11 & 19 & 0.064 & 0.694 & $0.78 \pm 0.40$ & $-24 \pm 22$ \\
$L_{r}-M_{\mathrm{H}_{2}}$ & all & 17 & 15 & -0.079 & 0.674 & $0.90 \pm 0.40$ & $-26 \pm 17$ \\
\hline
\end{tabular}

Notes. Column description: (1) relation studied; (2) sample considered; (3) number of sources with both quantities detected; (4) number of sources that show at least an upper limit in one of the two quantities; (5) the generalized Kendall's $\tau$, considering all the data; (6) $P_{\tau}$ probability associated with $\tau$ that the correlation is not present; (7)-(8) slope coefficient $m$ and intercept coefficient $q$ for the linear regression $(y=m * x+q)$.

the properties of the multiphase gas on the nuclear scale, instead of the galaxy scale, sets the accretion mode and the radio loudness of the AGN. In fact, at the center of low-luminosity radio galaxies (which include CoreG), on a scale of some parsecs, hot gas is known to be responsible for regulating the nuclear activity (e.g., Allen et al. 2006; Balmaverde et al. 2008; Nemmen \& Tchekhovskoy 2014).

Furthermore, we looked for a possible connection between the molecular gas content and the nuclear activity in [O III] line,
X-ray, optical, and radio bands. We did not find any highly significant correlations. However, the censored analysis indicates that trend between the $\mathrm{H}_{2}$ mass and the [O III] luminosity cannot be excluded. We interpret this link in terms of a possible increase in $\mathrm{CO}$ luminosity produced by heating of the molecular mass by the AGN radiation field, which also illuminates the narrowline region. This scenario still needs to be confirmed on a larger sample of RQ/RL AGN. This will be addressed to a forthcoming paper. Furthermore, it is fundamental to increase the number 
of $\mathrm{CO}$ detections to improve the statistics. The ALMA telescope certainly will play a decisive role in this field in the near future.

Acknowledgements. This work has benefited from research funding from the European Community's sixth Framework Program under RadioNet R113CT 2003 5058187. R.D.B. was supported at the Technion by a fellowship from the Lady Davis Foundation. We thank the referee for the constructive comments that have helped us to improve the paper.

\section{References}

Alatalo, K., Davis, T. A., Bureau, M., et al. 2013, MNRAS, 432, 1796

Allen, S. W., Dunn, R. J. H., Fabian, A. C., Taylor, G. B., \& Reynolds, C. S. 2006, MNRAS, 372, 21

Arimoto, N., Sofue, Y., \& Tsujimoto, T. 1996, PASJ, 48, 275

Baldi, R. D., \& Capetti, A. 2009, A\&A, 508, 603

Balmaverde, B., \& Capetti, A. 2006, A\&A, 447, 97

Balmaverde, B., Capetti, A., \& Grandi, P. 2006, A\&A, 451, 35

Balmaverde, B., Baldi, R. D., \& Capetti, A. 2008, A\&A, 486, 119

Bettoni, D., Galletta, G., \& García-Burillo, S. 2003, A\&A, 405, 5

Beuing, J., Dobereiner, S., Bohringer, H., \& Bender, R. 1999, MNRAS, 302, 209

Böker, T., Lisenfeld, U., \& Schinnerer, E. 2003, A\&A, 406, 87

Bolatto, A. D., Leroy, A. K., Rosolowsky, E., Walter, F., \& Blitz, L. 2008, ApJ, 686, 948

Boselli, A., Lequeux, J., \& Gavazzi, G. 2002, A\&A, 384, 33

Braine, J., \& Combes, F. 1992, A\&A, 264, 433

Braine, J., Combes, F., Casoli, F., et al. 1993, A\&AS, 97, 887

Burstein, D., Bender, R., Faber, S., \& Nolthenius, R. 1997, AJ, 114, 1365

Capetti, A., \& Balmaverde, B. 2005, A\&A, 440, 73

Capetti, A., \& Balmaverde, B. 2006, A\&A, 453, 27

Capetti, A., \& Balmaverde, B. 2007, A\&A, 469, 75

Casasola, V., Combes, F., Bettoni, D., \& Galletta, G. 2007, A\&A, 473, 771

Chiaberge, M., Capetti, A., \& Celotti, A. 1999, A\&A, 349, 77

Combes, F., Young, L. M., \& Bureau, M. 2007, MNRAS, 377, 1795

Crocker, A. F., Bureau, M., Young, L. M., \& Combes, F. 2011, MNRAS, 410, 1197

Davis, T. A., Alatalo, K., Bureau, M., et al. 2013, MNRAS, 429, 534

Fabbiano, G. 1989, ARA\&A, 27, 87

Faber, S. M., Tremaine, S., Ajhar, E. A., et al. 1997, AJ, 114, 1771

Fanaroff, B. L., \& Riley, J. M. 1974, MNRAS, 167, 31P

Feigelson, E. D., \& Nelson, P. I. 1985, ApJ, 293, 192

Glover, S. C. O., \& Mac Low, M.-M. 2011, MNRAS, 412, 337

Huchtmeier, W. K., Bregman, J. N., Hogg, D. E., \& Roberts, M. S. 1994, A\&A, 281,327

Isobe, T., Feigelson, E. D., \& Nelson, P. I. 1986, ApJ, 306, 490

Isobe, T., Feigelson, E. D., Akritas, M. G., \& Babu, G. J. 1990, ApJ, 364, 104
Israel, F. P. 1997, A\&A, 328, 471

Kaplan, E. L., \& Meier, P. 1958, Nonparametric estimation from incomplete observations, J. Am. Stat. Assoc., 457, 53

Kendall, M. 1983, Biometrika, 30, 81

Kharb, P., Capetti, A., Axon, D. J., et al. 2012, AJ, 143, 78

Knapp, G. R., Turner, E. L., \& Cunniffe, P. E. 1985, AJ, 90, 454

Knapp, G. R., Guhathakurta, P., Kim, D., \& Jura, M. A. 1989, ApJS, 70, 329

Komugi, S., Sofue, Y., Kohno, K., et al. 2008, ApJS, 178, 225

Lavalley, M., Isobe, T., \& Feigelson, E. 1992, in ASP Conf. Ser., 25, Astronomical Data Analysis Software and Systems I, eds. D. M. Worrall, C. Biemesderfer, \& J. Barnes, 245

Lees, J. F., Knapp, G. R., Rupen, M. P., \& Phillips, T. G. 1991, ApJ, 379, 177

Lisenfeld, U., Mundell, C. G., Schinnerer, E., Appleton, P. N., \& Allsopp, J. 2008, ApJ, 685, 181

Magrini, L., Bianchi, S., Corbelli, E., et al. 2011, A\&A, 535, A13

Maiolino, R., Ruiz, M., Rieke, G. H., \& Papadopoulos, P. 1997, ApJ, 485, 552

Maloney, P. 1990, in The Interstellar Medium in Galaxies, eds. H. A. Thronson Jr., \& J. M. Shull, Astrophys. Space Sci. Lib., 161, 493

Nemmen, R. S., \& Tchekhovskoy, A. 2014, MNRAS, submitted [arXiv: 1406.7420 ]

Nesvadba, N. P. H., Boulanger, F., Salomé, P., et al. 2010, A\&A, 521, A65

Ocaña Flaquer, B., Leon, S., Combes, F., \& Lim, J. 2010, A\&A, 518, A9

Ogle, P., Boulanger, F., Guillard, P., et al. 2010, ApJ, 724, 1193

O’Sullivan, E., Combes, F., Hamer, S., et al. 2015, A\&A, 573, A111

Papadopoulos, P. P., \& Seaquist, E. R. 1998, ApJ, 492, 521

Peto, R., \& Peto, J. 1972, Asymptotically efficient rank invariant procedures, J. Roy. Statistic. Soc., 135, 185

Phillips, M. M., Jenkins, C. R., Dopita, M. A., Sadler, E. M., \& Binette, L. 1986, AJ, 91, 1062

Sadler, E. M. 1987, in Structure and Dynamics of Elliptical Galaxies, ed. P. T. de Zeeuw, IAU Symp., 127, 125

Sage, L. J., \& Wrobel, J. M. 1989, ApJ, 344, 204

Sage, L. J., Welch, G. A., \& Young, L. M. 2007, ApJ, 657, 232

Schmitt, J. H. M. M. 1985, ApJ, 293, 178

Sofue, Y., \& Wakamatsu, K. 1993, PASJ, 45, 529

Solomon, P. M., \& Barrett, J. W. 1991, in Dynamics of Galaxies and Their Molecular Cloud Distributions, eds. F. Combes, \& F. Casoli, IAU Symp., 146, 235

Taniguchi, Y., Murayama, T., Nakai, N., Suzuki, M., \& Kameya, O. 1994, AJ, 108,468

Vollmer, B., Braine, J., Combes, F., \& Sofue, Y. 2005, A\&A, 441, 473

Welch, G. A., \& Sage, L. J. 2003, ApJ, 584, 260

Werner, N., Oonk, J. B. R., Sun, M., et al. 2014, MNRAS, 439, 2291

Wiklind, T., Combes, F., \& Henkel, C. 1995, A\&A, 297, 643

Wilson, A. S., \& Colbert, E. J. M. 1995, ApJ, 438, 62

Wrobel, J. M., \& Heeschen, D. S. 1991, AJ, 101, 148

Young, L. M. 2002, AJ, 124, 788

Young, L. M. 2005, ApJ, 634, 258

Young, L. M., Bureau, M., Davis, T. A., et al. 2011, MNRAS, 414, 940 